\title{
Metal chaperones: a holistic approach to the treatment of Alzheimer's disease
}

\section{Paul Anthony Adlard* and Ashley lan Bush}

The Mental Health Research Institute, University of Melbourne, Parkville, VIC, Australia

Edited by:

Lary C. Walker, Emory University, USA

Reviewed by:

Lary C. Walker, Emory University, USA Salvatore Oddo, University of Texas

Health Science Center, USA

\section{${ }^{*}$ Correspondence:}

Paul Anthony Adlard, Mental Health Research Institute, University of Melbourne, Kenneth Myer Building, At Genetics Lane on Royal Parade, Parkville, Victoria 3010, Australia. e-mail:padlard@unimelb.edu.au
As evidence for the role of metal ion dysregulation in the pathogenesis of multiple CNS disorders grows, it has become important to more precisely identify and differentiate the biological effects of various pharmacological modulators of metal ion homeostasis. This is particularly evident in disorders such as Alzheimer's disease (AD), where the use of metal chaperones (that transport metals), as opposed to chelators (which exclude metals from biological interactions), may prove to be the first truly disease modifying approach for this condition. The purpose of this mini-review is to highlight the emerging notion that metal chaperones, such as PBT2 (Prana Biotechnology), modulate a variety of critical pathways affecting key aspects of the AD cascade to provide a more "holistic" approach to the treatment of this disease.

Keywords: metal, Alzheimer's disease, therapeutic, cohaperone, PBT2

\section{INTRODUCTION}

The existing treatment options for mild to moderate Alzheimer's disease $(\mathrm{AD})$ patients are limited to a small number of FDAapproved pharmaceuticals, including the acetylcholinesterase inhibitors (AChEIs) Tacrine, Donepezil, Galantamine, and Rivastigmine. These compounds prevent the degradation of acetylcholine (AChE) and consequently increase the concentrations of this neurotransmitter in the synaptic cleft to ultimately enhance cholinergic neurotransmission (Lleo et al., 2006). A more recently approved medication is the $N$-methyl-D-aspartate (NMDA)-receptor antagonist Memantine, which protects against glutamate excitotoxicity (Hynd et al., 2004; Sonkusare et al., 2005; Parsons et al., 2007) and also inhibits pathological reactions of the tau protein (such as hyperphosphorylation and aggregation; Li et al., 2004). The important caveat for all of these treatment options is that none of them afford significant long-term symptomatic relief let alone provide hope for a cure. To address this, there are numerous compounds currently in various phases of clinical trial, and even more in preclinical development, which are believed may offer some hope for a tangible clinical benefit in $\mathrm{AD}$. We have recently published an extensive review of these compounds (Biran et al., 2009), the majority of which target one or both of the primary aggregating proteins implicated in the pathogenesis of $\mathrm{AD}$.

\section{RECENT CLINICAL FAILURES IN AD}

While there are a multitude of compounds currently in clinical trial that focus on a variety of cellular targets, the majority have been developed around the principles of the amyloid cascade hypothesis (Adlard et al., 2009). It has come to pass, however, that many of the compounds that were heralded as the shining light of $\mathrm{AD}$ therapeutics have stumbled in the final phases of clinical testing, casting a shadow over the field (Golde et al., 2011).

Principle among the high profile failures have been; Tramiprosate (Alzhemed), an inhibitor of $A \beta$ oligomerization;
Tarenflurbil (Flurizan), a gamma-secretase modulator; Semagacestat (LY450139 Dihydrate), a gamma-secretase inhibitor; and Latrepirdine (Dimebon), which has a plethora of biological effects, but whose main biological efficacy may derive from an effect on mitochondria. These have formed the basis for several commentaries (Karran et al., 2011; Sperling et al., 2011) on the state of AD clinical trials and therapeutics, and will not be discussed here. What these failed trials do suggest, however, is that compounds whose mechanism of action is to solely target the beta-amyloid protein, and hence which can be considered to be "classical approaches" (Masters and Beyreuther, 2006), may not represent the optimal approach to the treatment of AD. Compounds that provide greater and longer-lived symptomatic relief or which represent more global/holistic approaches by targeting a number of key aspects of the disease process may represent better drug candidates than the classical approaches. To this end, there is ever-increasing evidence for the efficacy of metal chaperones in the treatment of AD (Duce et al., 2011).

\section{METAL CHAPERONES}

Metal chaperones (or metallochaperones) are compounds that function to shuttle metal ions to specific intracellular target proteins. This facilitation of metal transport is distinct from metal chelators or buffers, which function to exclude or deplete metals from discrete cellular compartments to thereby limit biological interactions of key metal ions. Cumulatively, however, these processes serve to maintain tight regulatory control over cellular metal ion homeostasis such that the intracellular concentration of freely available metal ions (such as copper and zinc) is close to zero.

The components involved in these processes include transporters (such as CTR1 for copper and ZIP for zinc), channels (such as voltage gated calcium channels), and pumps (such as calcium-ATPase), which allow the transport of metal ions across biological membranes; endocytosis of protein:metal complexes 
(such as transferrin:iron) into the cell and chaperones (such as CCS for copper), which escort metals into the cell. These, and other, processes involved in the highly complex process of metal trafficking and homeostasis have been critically reviewed elsewhere (Ba et al., 2009) and will not be discussed here.

Such a high level of control at many cellular "levels" is essential in limiting potentially deleterious interactions of redox active transition metal ions, which are implicated in the pathogenesis of a number of disorders including AD (Sayre et al., 1999; Smith et al., 2007). The involvement of metal ions in disease extends the breadth from being involved in creating an adverse cellular milieu (which among other things, may promote cell death through the activation of particular pathways that lead to degeneration) through to direct involvement in the generation and toxicity of the principle toxic moiety in diseases such as $\mathrm{AD}$.

Given the compelling evidence for the critical role of copper and zinc in both precipitating and potentiating AD (Adlard and Bush, 2006; Adlard et al., 2009; Duce et al., 2011), attempts to modulate the metal:AD interaction are an emerging therapeutic strategy. Genetic studies in various mouse models of $\mathrm{AD}$ supports this notion. Lee et al. (2002), for example, demonstrated that the ablation of the synaptic zinc transporter [zinc transporter 3 (ZnT3); Palmiter et al., 1996; Cole et al., 1999] in Tg2576 mice [a mutant human amyloid precursor protein (APP) over-expressing $\mathrm{AD}$ mouse model] resulted in a loss of vesicular zinc in glutamatergic synapses and a concomitant reduction in both $A \beta$ plaques (number and/or percentage area occupied within the brain) and insoluble $A \beta$ burden. These data are supported by more recent reports that have clearly defined a role for zinc in synaptic pathways critically affected in AD (Adlard et al., 2010), and indeed, the 3xTg mouse model of AD has shown a gender-dependent alteration in vesicular zinc-related plasticity (Nakashima et al., 2010). Deshpande et al. (2009) have also demonstrated that $A \beta$ oligomers are attracted to zinc emanating from the glutamatergic synapse, and that this can then occlude the NMDAR2B receptor and thus affect downstream signaling pathways. In another study, by Phinney et al. (2003), TgCRND8 mice (a double mutant human APP over-expressing AD mouse model) were crossed with $t x^{\mathrm{J}}$ mice to generate mice with a mutation in the transport protein (ATPase7b) that loads copper into secretory vesicles (Lutsenko and Petris, 2003). This resulted in elevated brain copper levels and a concomitant significant reduction in the number of dense cored plaques in the cortex and hippocampus.

While such genetic approaches are unlikely to become a therapeutic option for the treatment of $\mathrm{AD}$, it does highlight the relevance of targeting metal ions in this disease. In addition to the modulation of endogenous factors such as these and others (such as metallothioneins), there have been numerous attempts at modulating metal levels within the CNS through dietary modification and pharmacological intervention.

Utilizing the APP23 mouse line (a mutant human APP overexpressing AD mouse model), Bayer et al. (2003) demonstrated that 3 months of elevated dietary copper intake was sufficient to remediate the copper deficit present in the APP23 mouse model, and to also lower $\mathrm{A} \beta$ burden. Similarly, increasing the dietary intake of zinc in both the TgCRND8 (for 5 months) and Tg2576 (for 11 months) mice (Linkous et al., 2009) resulted in a reduction in $\mathrm{A} \beta$ burden and a histological reduction in plaque size (which was also paradoxically associated with impaired performance on behavioral assessments of cognition). These data are consistent with earlier work (Stoltenberg et al., 2007) demonstrating that mice over-expressing mutant human APP and Presenilin 1 (PS1; APP/PS1) maintained on a zinc-deficient diet for 3 months had a significant elevation in $A \beta$ plaque burden and a histological increase in plaque size.

Cumulatively, these data highlight the dynamic nature of $\mathrm{AD}$ like pathology in the various mouse models and importantly, identify a critical role for metal ion homeostasis in the pathogenesis of disease. This complexity, however, is exacerbated by the apparent interaction between metals such as copper and zinc. A recent study (Railey et al., 2011), for example, demonstrated that the cognitive deficits observed in zinc-supplemented mouse models of $\mathrm{AD}$ can be overcome by parallel copper supplementation. Furthermore, high levels of dietary zinc can inhibit the intestinal absorption of copper (Sandstead, 1995) and lower cortical copper levels (Flinn et al., 2005), while Wilson's disease (a copper-overload disorder) is effectively treated with dietary zinc (Sinha and Taly, 2008). Thus, any biological effects derived from the modulation of a particular metal species may, in fact, be affected by a consequent alteration in a different metal ion.

These data also suggest that a singular mechanistic approach is unlikely to afford the long-term benefit required for a truly effective and disease modifying therapeutic - as in the brief discussion above, it is clear that homeostatic deficits in metal ions need to be rectified, but at the same time, the elimination of metals from discrete cellular targets/zones (e.g., the synapse) also modifies the progression of disease [but this may come at a cognitive cost - as is implied from the data showing that reductions in synaptic zinc levels reduce plaque burden in $\mathrm{Tg}$ mice (Lee et al., 2002), but also result in severe cognitive deficits when ablated in WT animals (Adlard et al., 2010); the cognitive effect of ZnT3 ablation in $\mathrm{Tg}$ mice has yet to be assessed]. Thus, highly targeted approaches that either increase or decrease a particular metal ion may be inherently confounded. In this regard, metal chaperones (which have also variously been referred to as "ionophores" and "metal-protein attenuating compounds") may represent the "sweet spot" of metal-targeted therapeutics for $\mathrm{AD}$ because they foster the maintenance and/or restoration of metal ion homeostasis which then impacts a raft of "healthy" and "pathological" cellular pathways that ultimately promotes "normal" function. Such context-dependent modulation of metal levels may prove critical for long-term therapeutic strategies that target metal ions.

\section{METAL CHAPERONES: A NOVEL THERAPEUTIC STRATEGY FOR AD?}

A number of metal-targeted approaches have been employed in both preclinical animal studies and human clinical trials, and we have critically reviewed these recently (Duce et al., 2011). However, one of the more promising chaperone candidates that has 
an evolving literature, particularly surrounding its mechanism of action, is PBT2 (Prana Biotechnology).

Utilizing both Tg2576 and APP/PS1 animals, we have previously demonstrated that varying PBT2 treatment paradigms (acute and chronic dosing) result in significant decreases in histological $A \beta$ plaque burden, a significant decrease in biochemical $A \beta$ load and a significant decrease in interstitial fluid $A \beta$ levels (Adlard et al., 2008). Further to this, the other side of the "Alzheimer's equation" is the loss of functional tau resulting from its hyperphosphorylation (and subsequent formation of intracellular neurofibrillary tangles), and to this end, we have demonstrated that PBT2 significantly decreases the levels of phosphorylated tau in animal models of AD (Adlard et al., 2008). Thus, the compound has the potential to modulate both major hallmark pathologies in the $\mathrm{AD}$ brain. These pathologies, while central to the clinical diagnosis of $\mathrm{AD}$ and also reported to be critical to the pathogenesis of disease, are ultimately important more for their downstream effects on normal brain function/cellular pathways. In this regard, $\mathrm{AD}$ has often been referred to as a disease of disconnection, where the loss of synaptic connections gradually erodes memory and other higher order cognitive functions to drive the symptomatic presentation of disease (potentially as a direct consequence of the deposition of $A \beta$ ). If a therapeutic were to have an effect on $A \beta$ or tau in the absence of any subsequent downstream improvement in critical disease measures (e.g., memory) and their associated anatomical correlates (e.g., synapses and dendritic spines), then the approach would be fundamentally limited and not provide any level of long-term disease modification. In this respect, we have demonstrated that PBT2 significantly increases synaptophysin levels (a surrogate marker for synapses) in transgenic mouse models of $\mathrm{AD}$ (Adlard et al., 2008), in addition to restoring hippocampal dendritic spine density in both young and old transgenic mouse models of AD (Adlard et al., 2011). These effects occurred in conjunction with a restoration in the levels of key synaptic proteins involved in cognition, such as NMDA receptors as well as elements of the BDNF pathway. Preliminary in vitro evidence supported the notion that PBT2 acts in a metal-dependent manner to regulate neurite outgrowth and NMDA receptor protein levels (Adlard et al., 2011), and this has been further defined in a more recent study examining the mechanisms of action of PBT2 (Crouch et al., 2011), as described below.

PBT2 treatment of SH-SY5Y cells has been shown to result in a metal-dependent phosphorylation of glycogen synthase kinase 3 (GSK; which may occur via an inhibition of the phosphatase calcineurin, whose other substrates such as CREB and CaMKII are also modulated by PBT2) which subsequently inhibits the activity of GSK, one of the major tau kinases in the brain (Lei et al., 2011). Importantly, the metals translocated into the cell by PBT2, which can also activate numerous other cellular pathways involved in neuronal and synaptic health, can be effectively "sourced" from protease-resistant extracellular A $\beta: Z n$ aggregates, which themselves then become subject to degradation by endogenous clearance mechanisms such as matrix metalloproteases after the metal is removed from the $A \beta$. This highlights the biological relevance of the activity of $\mathrm{PBT} 2$, and also clearly demonstrates the notion that PBT2 has multiple metal-dependent activities that affect several different key aspects of the AD cascade, including the triad of $A \beta$, tau and cognition.

Cumulatively, these specific effects on disease-related pathways and endpoints are likely to represent the major underlying mechanism for the rapid PBT2-dependent reversal of the cognitive impairment observed in vivo (Adlard et al., 2008). These in vitro and in vivo studies showing potential efficacy for this metal chaperone in targeting both biomarkers and the major symptomatic feature of disease are supported by an emerging clinical literature.

The safety and efficacy of PBT2 was assessed in a small [ $n=29$ placebo; $n=20$ low dose PBT2 (50 mg/day); $n=29$ high dose PBT2 $250 \mathrm{mg} /$ day] 12 week, double-blind and placebo-controlled trial (Lannfelt et al., 2008). The safety profile for PBT2 was favorable and it also showed a significant modulation of biomarkers and clinical endpoints. Specifically, PBT2 treatment significantly decreased CSF levels of $A \beta_{1-42}$ in a dose-dependent manner, as compared to the placebo group. Cognitive testing, which included ADAS-cog, MMSE and a neuropsychological test battery (NTB), revealed that the high dose treatment group had a significant improvement in executive function compared to the placebo-treated group. Further analysis of the cognitive data (Faux et al., 2010) revealed that, based upon a ranking analysis, the high dose treatment group had a significantly higher proportion of patients showing improvement on the NTB composite $z$-score and executive factor $z$-score as compared to the placebo group, with the ADAS-cog data also approaching significance $(p=0.056)$.

There are ongoing AD trials with PBT2, with a 12 month Phase II imaging trial currently recruiting. This study is designed to assess the anatomical correlates of PBT2 treatment, specifically looking at the effect of PBT2 on A $\beta$ plaque load in the living human brain, as well as to consolidate the evidence for longer-term cognitive benefits of this compound (www.pranabio.com). Much larger Phase III trials will then be required to definitively establish the efficacy of PBT2 for the treatment of AD. This is a significant hurdle for any new compound to pass, and only in testing will it be established if PBT2 is any different to the other promising Phase II candidates that have not met expectations in Phase III trials.

\section{CONCLUSION}

Taken together, these data provide compelling evidence for the efficacy of metal-targeted approaches in the treatment of $\mathrm{AD}$, and also specifically point to the use of metal chaperones as being one particularly effective strategy. In this review we have highlighted the reported activities and potential for PBT2, an 8-hydroxy quinoline compound. A recent screen of 200,000 compounds in a yeast model system identified this class of compound as being particularly effective at preventing proteotoxicity. It was also identified that different 8-hydroxy quinolines exhibited distinct activities on metal ion homeostasis and metalloprotein activities (subtle changes to the molecular backbone evinced significant changes in biological activity), implicating the therapeutic potential for different 8-hydroxy quinolines against a range of different 
neurodegenerative disorders (Tardiff et al., 2012). Thus, while metal chaperones such as PBT2 may prove efficacious in the treatment of $\mathrm{AD}$ and a host of other disorders that are characterized by metal ion dyshomeostasis, it will remain crucial to identify the optimal metal chaperone for a given disorder, and to also treat at the right stage of disease.

\section{REFERENCES}

Adlard, P. A., Bica, L., White, A. R., Nurjono, M., Filiz, G., Crouch, P. J., Donnelly, P. S., Cappai, R., Finkelstein, D. I., and Bush, A. I. (2011). Metal ionophore treatment restores dendritic spine density and synaptic protein levels in a mouse model of Alzheimer's disease. PLoS ONE 6, e17669. doi:10.1371/journal.pone. 0017669

Adlard, P. A., and Bush, A. I. (2006). Metals and Alzheimer's disease. J. Alzheimers Dis. 10, 145-163.

Adlard, P. A., Cherny, R. A., Finkelstein, D. I., Gautier, E., Robb, E., Cortes, M., Volitakis, I., Liu, X., Smith, J. P., Perez, K., Laughton, K., Li, Q. X., Charman, S. A., Nicolazzo, J. A., Wilkins, S., Deleva, K., Lynch, T., Kok, G., Ritchie, C. W., Tanzi, R. E., Cappai, R., Masters, C. L., Barnham, K. J., and Bush, A. I. (2008). Rapid restoration of cognition in Alzheimer's transgenic mice with 8-hydroxy quinoline analogs is associated with decreased interstitial Abeta. Neuron 59, 43-55.

Adlard, P. A., James, S. A., Bush, A. I., and Masters, C. L. (2009). Beta-amyloid as a molecular therapeutic target in Alzheimer's disease. Drugs Today 45, 293-304.

Adlard, P. A., Parncutt, J. M., Finkelstein, D. I., and Bush, A. I. (2010). Cognitive loss in zinc transporter3 knock-out mice: a phenocopy for the synaptic and memory deficits of Alzheimer's disease? J. Neurosci. 30, 1631-1636.

Ba, L. A., Doering, M., Burkholz, T., and Jacob, C. (2009). Metal trafficking: from maintaining the metal homeostasis to future drug design. Metallomics 1, 292-311.

Bayer, T. A., Schafer, S., Simons, A., Kemmling, A., Kamer, T., Tepest, R., Eckert, A., Schüssel, K., Eikenberg, O., Sturchler-Pierrat, C., Abramowski, D., Staufenbiel, M., and Multhaup, G. (2003). Dietary $\mathrm{Cu}$ stabilizes brain superoxide dismutase 1 activity and reduces amyloid Abeta production in APP23 transgenic mice. Proc. Natl. Acad. Sci. U.S.A. 100, 14187-14192.

Biran, Y., Masters, C. L., Barnham, K. J., Bush, A. I., and Adlard, P.
A. (2009). Pharmacotherapeutic targets in Alzheimer's disease. J. Cell. Mol. Med. 13, 61-86.

Cole, T. B., Wenzel, H. J., Kafer, K. E., Schwartzkroin, P. A., and Palmiter, R. D. (1999). Elimination of zinc from synaptic vesicles in the intact mouse brain by disruption of the ZnT3 gene. Proc. Natl. Acad. Sci. U.S.A. 96, 1716-1721.

Crouch, P. J., Savva, M. S., Hung, L. W., Donnelly, P. S., Mot, A. I., Parker, S. J., Greenough, M. A., Volitakis, I., Adlard, P. A., Cherny, R. A., Masters, C. L., Bush, A. I., Barnham, K. J., and White, A. R. (2011). The Alzheimer's therapeutic PBT2 promotes amyloid-beta degradation and GSK3 phosphorylation via a metal chaperone activity. J. Neurochem. 119, 220-230.

Deshpande, A., Kawai, H., Metherate, R., Glabe, C. G., and Busciglio, J. (2009). A role for synaptic zinc in activitydependent Abeta oligomer formasynapses. J. Neurosci. 29, 4004-4015.

Duce, J. A., Bush, A. I., and Adlard, P. A. (2011). Role of amyloid-b-metal interactions in Alzheimer's disease. Future Neurol. 6, 641-659.

Faux, N. G., Ritchie, C. W., Gunn, A., Rembach, A., Tsatsanis, A., Bedo, J., Harrison, J., Lannfelt, L., Blennow, K., Zetterberg, H., Ingelsson, M., Masters, C. L., Tanzi, R. E., Cummings, J. L., Herd, C. M., and Bush, A. I. (2010). PBT2 rapidly improves cognition in Alzheimer's disease: additional phase II analyses. J. Alzheimers Dis. 20, 509-516.

Flinn, J. M., Hunter, D., Linkous, D. H., Lanzirotti, A., Smith, L. N., Brightwell, J., and Jones, B. F. (2005). Enhanced zinc consumption causes memory deficits and increased brain levels of zinc. Physiol. Behav. 83, 793-803.

Golde, T. E., Schneider, L. S., and Koo, E. H. (2011). Anti-abeta therapeutics in Alzheimer's disease: the need for a paradigm shift. Neuron 69, 203-213.

Hynd, M. R., Scott, H. L., and Dodd, P. R. (2004). Glutamate-mediated exciAlzheimer's disease. Neurochem. Int. $45,583-595$. tion and accumulation at excitatory totoxicity and neurodegeneration in

\section{ACKNOWLEDGMENTS}

Paul Anthony Adlard and Ashley Ian Bush are shareholders in Prana Biotechnology, which had no input into the preparation of this manuscript. Paul Anthony Adlard and Ashley Ian Bush are supported by grants from the NHMRC, ARC, Alzheimer's Association (USA) and Clemenger Trust.

Karran, E., Mercken, M., and De Strooper, B. (2011). The amyloid cascade hypothesis for Alzheimer's disease: an appraisal for the development of therapeutics. Nat. Rev. Drug Discov. 10, 698-712.

Lannfelt, L., Blennow, K., Zetterberg, H. Batsman, S., Ames, D., Harrison, J. Masters, C. L., Targum, S., Bush, A. I., Murdoch, R., Wilson, J., Ritchie, C. W., and PBT2-201-EURO Study Group. (2008). Safety, efficacy, and biomarker findings of PBT2 in targeting Abeta as a modifying therapy for Alzheimer's disease: a phase IIa, double-blind, randomised, placebocontrolled trial. Lancet Neurol. 7 , 779-786.

Lee, J. Y., Cole, T. B., Palmiter, R. D. Suh, S. W., and Koh, J. Y. (2002). Contribution by synaptic zinc to the gender-disparate plaque formation in human Swedish mutant APP transgenic mice. Proc. Natl. Acad. Sci. U.S.A. 99, 7705-7710.

Lei, P., Ayton, S., Bush, A. I., and Adlard, P. A. (2011). GSK-3 in neurodegenerative diseases. Int. J. Alzheimer's Dis. 2011, 189246.

Li, L., Sengupta, A., Haque, N., Grundke-Iqbal, I., and Iqbal, K. (2004). Memantine inhibits and reverses the Alzheimer type abnormal hyperphosphorylation of tau and associated neurodegeneration. FEBS Lett. 566, 261-269.

Linkous, D. H., Adlard, P. A., Wanschura, P. B., Conko, K. M., and Flinn, J. M. (2009). The effects of enhanced zinc on spatial memory and plaque formation in transgenic mice. J. Alzheimers Dis. 18 565-579.

Lleo, A., Greenberg, S. M., and Growdon, J. H. (2006). Current pharmacotherapy for Alzheimer's disease. Annu. Rev. Med. 57, 513-533.

Lutsenko, S., and Petris, M. J. (2003). Function and regulation of the mammalian copper-transporting ATPases: insights from biochemical and cell biological approaches. J. Membr. Biol. 191, 1-12.

Masters, C. L., and Beyreuther, K. (2006). Alzheimer's centennial legacy: prospects for rational therapeutic intervention targeting the
Abeta amyloid pathway. Brain 129, 2823-2839.

Nakashima, A. S., Oddo, S., Laferla, F. M., and Dyck, R. H. (2010) Experience-dependent regulation of vesicular zinc in male and female 3xTg-AD mice. Neurobiol. Aging 31, 605-613.

Palmiter, R. D., Cole, T. B., Quaife, C. J., and Findley, S. D. (1996). ZnT3 , a putative transporter of zinc into synaptic vesicles. Proc. Natl. Acad. Sci. U.S.A. 93, 14934-14939.

Parsons, C. G., Stoffler, A., and Danysz, W. (2007). Memantine: a NMDA receptor antagonist that improves memory by restoration of homeostasis in the glutamatergic system too little activation is bad, too much is even worse. Neuropharmacology 53, 699-723.

Phinney, A. L., Drisaldi, B., Schmidt, S. D., Lugowski, S., Coronado, V., Liang, Y., Horne, P., Yang, J., Sekoulidis, J., Coomaraswamy, J., Chishti, M. A., Cox, D. W., Mathews, P. M., Nixon, R. A., Carlson, G. A., St. George-Hyslop, P., and Westaway, D. (2003) In vivo reduction of amyloid-beta by a mutant copper transporter. Proc. Natl. Acad. Sci. U.S.A. 100, 14193-14198.

Railey, A. M., Groeber, C. M., and Flinn, J. M. (2011). The effect of metals on spatial memory in a transgenic mouse model of Alzheimer's disease. J. Alzheimers Dis. 24 , 375-381.

Sandstead, H. H. (1995). Requirements and toxicity of essential trace elements, illustrated by zinc and copper. Am. J. Clin. Nutr. 61, 621S-624S.

Sayre, L. M., Perry, G., and Smith, M. A. (1999). Redox metals and neurodegenerative disease. Curr. Opin. Chem. Biol. 3, 220-225.

Sinha, S., and Taly, A. B. (2008). Withdrawal of penicillamine from zinc sulphate-penicillamine maintenance therapy in Wilson's disease: promising, safe and cheap. J. Neurol. Sci. 264, 129-132.

Smith, D. G., Cappai, R., and Barnham, K. J. (2007). The redox chemistry of the Alzheimer's disease amyloid beta peptide. Biochim. Biophys. Acta 1768, 1976-1990. 
Sonkusare, S. K., Kaul, C. L., and Ramarao, P. (2005). Dementia of Alzheimer's disease and other neurodegenerative disorders - memantine, a new hope. Pharmacol. Res. 51, $1-17$.

Sperling, R. A., Jack, C. R. Jr., and Aisen, P. S. (2011). Testing the right target and right drug at the right stage. Sci. Transl. Med. 3, $111 \mathrm{~cm} 133$.

Stoltenberg, M., Bush, A. I., Bach, G., Smidt, K., Larsen, A., Rungby J, Lund S, Doering P, Danscher
G. (2007). Amyloid plaques arise from zinc-enriched cortical layers in APP/PS1 transgenic mice and are paradoxically enlarged with dietary zinc deficiency. Neuroscience 150, 357-369.

Tardiff, D. F., Tucci, M. L., Caldwell, K. A., Caldwell, G. A., and Lindquist, S. (2012). Different 8-hydroxyquinolines protect models of TDP-43, alpha-synuclein, and polyglutamine proteotoxicity through distinct mechanisms. J. Biol. Chem. 287, 4107-4120.
Conflict of Interest Statement: The authors declare that the research was conducted in the absence of any commercial or financial relationships that could be construed as a potential conflict of interest.

Received: 29 December 2011; paper pending published: 25 January 2012; accepted: 14 February 2012; published online: 02 March 2012.

Citation: Adlard PA and Bush AI (2012) Metal chaperones: a holistic approach to the treatment of Alzheimer's disease. Front. Psychiatry 3:15. doi: 10.3389/fpsyt.2012.00015

This article was submitted to Frontiers in Neurodegeneration, a specialty of Frontiers in Psychiatry.

Copyright (c) 2012 Adlard and Bush. This is an open-access article distributed under the terms of the Creative Commons Attribution Non Commercial License, which permits non-commercial use, distribution, and reproduction in other forums, provided the original authors and source are credited. 